\title{
Open and Minimally Invasive Transforaminal Lumbar Interbody Fusion: Comparison of Intermediate Results and Complications
}

\author{
Hwee Weng Dennis $\mathrm{Hey}^{1}$, Hwan Tak Hee ${ }^{2}$ \\ ${ }^{1}$ Department of Orthopaedic Surgery, National University Health System, Singapore \\ ${ }^{2}$ Centre for Spine and Scoliosis Surgery, Paramount and Mount Elizabeth Medical Centre, Singapore
}

\section{Study Design: Prospective study.}

Purpose: To compare clinical and radiological outcomes of open vs. minimally invasive transforaminal lumbar interbody fusion (MI-TLIF). Overview of Literature: MI-TLIF promises smaller incisions and less soft tissue dissection resulting in lower morbidity and faster recovery; however, it is technically challenging.

Methods: Twenty-five patients with MI-TLIF were compared with 25 matched open TLIF controls. A minimum 2 year follow-up and a statistical analysis of perioperative and long-term outcomes were performed. Potential complications were recorded.

Results: The mean ages for the open and MI-TLIF cases were 44.4 years (range, 19-69 years) and 43.6 years (range, 20-69 years), respectively. The male:female ratio was 13:12 for both groups. Average follow-up was 26.9 months for the MI-TLIF group and 29.3 months for the open group. Operative duration was significantly longer in the MI-TLIF group than that in the open group ( $p<0.05)$. No differences in estimated blood loss, duration to ambulation, or length of stay were found. Significant improvements in the Oswestry disability index and EQ-5D functional scores were observed at 6-, 12-, and 24-months in both groups, but no significant difference was detected between the groups. Fusion rates were comparable. Cage sizes were significantly smaller in the MI-TLIF group at the L5/S1 level ( $p<0.05)$. One patient had residual spinal stenosis at the MI-TLIF level, and one patient who underwent two-level MI-TLIF developed a deep vein thrombosis resulting in a pulmonary embolism.

Conclusions: MI-TLIF and open TLIF had comparable long-term benefits. Due to technical constraints, patients should be advised on the longer operative time and potential undersizing of cages at the L5S1 level.

Keywords: Duration of surgery; Interbody cage; Minimally invasive; Open; Oswestry disability index; Transforaminal lumbar interbody fusion

\section{Introduction}

Transforaminal lumbar interbody fusion (TLIF) is performed for conditions, including spinal stenosis, degenerative scoliosis, and spondylolisthesis of the lumbar spine [1]. It involves placing an interbody cage in an oblique direction and a strong pedicle screw posterior construct that temporarily stabilizes the spine until bony fusion takes place via sentinel and interbody bone grafting [2]. Potential dangers from mobilizing the thecal sac

Received May 29, 2014; Revised Jun 13, 2014; Accepted Jun 22, 2014

Corresponding author: Hey Hwee Weng Dennis

Department of Orthopaedic Surgery, National University Hospital, 1E Kent Ridge Road, NUHS Tower Block Level 11, Singapore 119228

Tel: +65-9620-1659, Fax: +65-6778-0720, E-mail: hweeweng@gmail.com 
and retracting the nerve roots are reduced compared to posterior lumbar interbody fusion techniques $[3,4]$. By removing motion between lumbar vertebral segments, TLIF alleviates axial mechanical pain that arises from instability and segmental degeneration of the lumbar spine. This approach also has the advantage of decompressing the cauda equina and exiting nerve root.

TLIF can be performed using an open approach or via a minimally invasive method (MI-TLIF). Open TLIF is associated with extensive dissection of paravertebral musculature and disruption of the natural posterior tension band effect from the interspinous and supraspinous ligaments leading to greater morbidity and slower recovery [5]. Thus, MI-TLIF is a viable alternative and has received increased interest. With the same fusion technique, MI-TLIF promises smaller skin incisions and less muscle dissection. In the early part of the learning curve, intraoperative technical difficulties are not infrequent [6]. The purpose of this study was to compare the clinical and radiological outcomes of open vs. MI-TLIF.

\section{Materials and Methods}

Twenty-five consecutive patients who underwent MITLIF, and 25 patients who underwent open TLIF performed at National University Hospital from 2006 to 2009 were match paired based on age, sex, level operated, indication for surgery, and period when surgery took place. All paired patients had a maximum age difference of 5 years and had their surgeries performed within 6 months of each other. All patients were operated on by a single fellowship trained surgeon with 8 years experience in lumbar spine surgery, who had performed more than 400 cases of open TLIFs prior to the start of this study. The MI-TLIF cases represented the early cases performed by this surgeon. All patients were offered either MI-TLIF or open TLIF as the same procedure but using different approaches. The smaller incision and dissection of the MI-TLIF technique were explained to all patients. Cost was the single overwhelming factor that resulted in patients selecting open TLIF compared to MI-TLIF, as there was a \$1,600 USD difference between MI-TLIF and open TLIF implants during the course of the study. The cost of implants is largely borne by patients in Singapore. Institutional Board Review was obtained for this study.

Pertinent information for this study was obtained from both electronic hospital data and manual case records.
Data included patient demographics (age and sex), indication for surgery (degenerative disc disease, prolapsed intervertebral discs, spinal stenosis, and spondylolisthesis), level(s) of surgery, intraoperative details (duration of surgery, estimated blood loss, type of implant), immediate postoperative parameters (decrease in hemoglobin, length of hospital stay, minimum time before ambulation), preoperative and postoperative long-term functional assessments (Oswestry disability index [ODI] and EQ-5D), and postoperative complications. Fusion rates were assessed based on the Bridwell classification [7].

Twenty-five subjects were required for each group based on the matched study design to achieve statistical significance with $80 \%$ power and a two-sided test of $5 \%$ for a $20 \%$ difference (standard deviation $=25 \%$ ) between the two groups based on the following outcomes: mean duration of surgery, estimated blood loss, decrease in hemoglobin, length of hospital stay, minimum time before ambulation, and ODI and EQ-5D scores.

Functional assessment questionnaires were completed by the patients with the help of independent interviewers before surgery and at 6 months, 1 year, and 2 years after surgery. The interviewers were blinded to the study. Fusion rates were assessed using plain radiographic anteroposterior and lateral projections of the lumbar spine at 2 years. They were interpreted by two spine surgeons and a radiologist not involved in the direct care of the patients. The final fusion grade (Bridwell classification) [7] was computed from the average of the three grades awarded by the three assessors. Although the best method to assess fusion was computed tomography, this was not possible due to cost and radiation issues. All patients were followed up for a minimum of 2 years after surgery.

Data entry was performed using a spreadsheet application (Excel 2003, Microsoft Co., Redmond, WA, USA). The statistical analysis was performed using SPSS ver. 16 (SPSS Inc., Chicago, IL, USA). Means of continuous variables were assessed using the $t$-test, and categorical variables were evaluated using the chi-square test. Statistical significance was defined as $p<0.05$.

\section{MI-TLIF technique}

The MI-TLIF procedure was performed with the patient placed in the prone position on a Jackson or Amsco operating table. A para-midline incision of approximately $2.5 \mathrm{~cm}$ was made using fluoroscopic guidance to locate 
the medial border of the facet joint. The erector spinae muscles were retracted until a probe was allowed to dock on the relevant facet joint. This was followed by insertion of serial dilators until a self-retaining retractor was assembled (Figs. 1, 2).

Fluoroscopy was used to confirm correct positioning and trajectory of the retractor. This step was important, as smooth execution of the rest of the procedure (except pedicle screw placement) hinges on correct trajectory of the retractor because the surgeon needs to operate within the confines of the retractor (Figs. 3, 4).

The surgical techniques of decompression and interbody fusion were similar for both the open and MI-TLIF cases. Facetectomy and annulotomy were performed to allow access to the intervertebral disc. This was followed by discectomy and preparation of the adjacent vertebral endplates. The disc space was distracted sequentially to

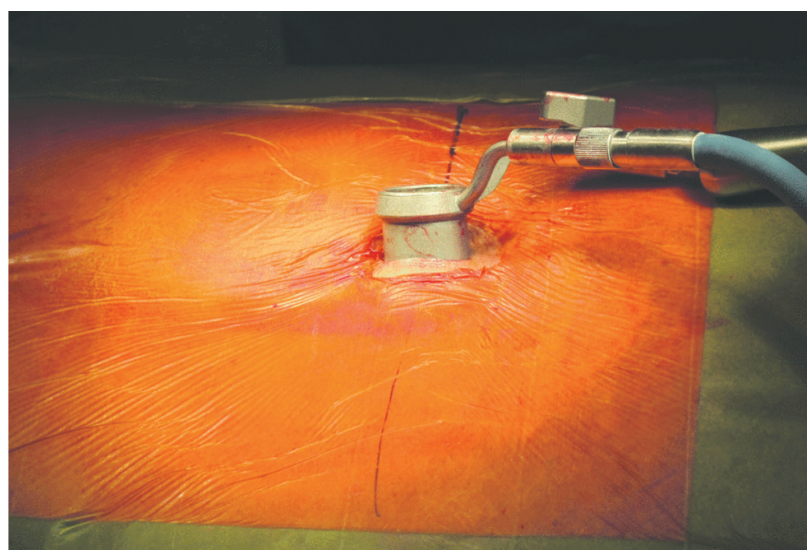

Fig. 1. Minimally invasive posterior para-midline approach to the lumbar spine using tubular retractors.

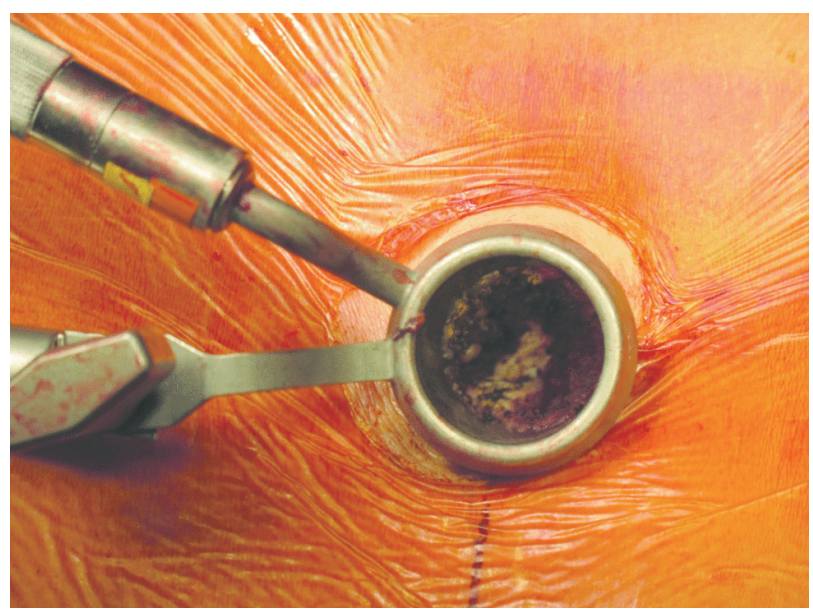

Fig. 2. Identifying the lamina bone using tubular retractors. the appropriate height, and a trial cage was tested before inserting the actual cage packed with a morcelised local bone graft. Final placement of the cage was confirmed fluoroscopically. A sentinel bone graft was placed anterior to the interbody cage.

The center of the pedicle was verified using fluoroscopic guidance with a true anteroposterior view, and the spinous process was centered between the two pedicles. The superior endplate of the vertebra was flat. Subsequently, two stab incisions (for single level MI-TLIF) of approximately $1.5 \mathrm{~cm}$ were made over the contralateral side. Jamshidi needles were inserted via these incisions on the contralateral side and the para-midline incision on the ipsilateral side. The needles were docked on the lateral

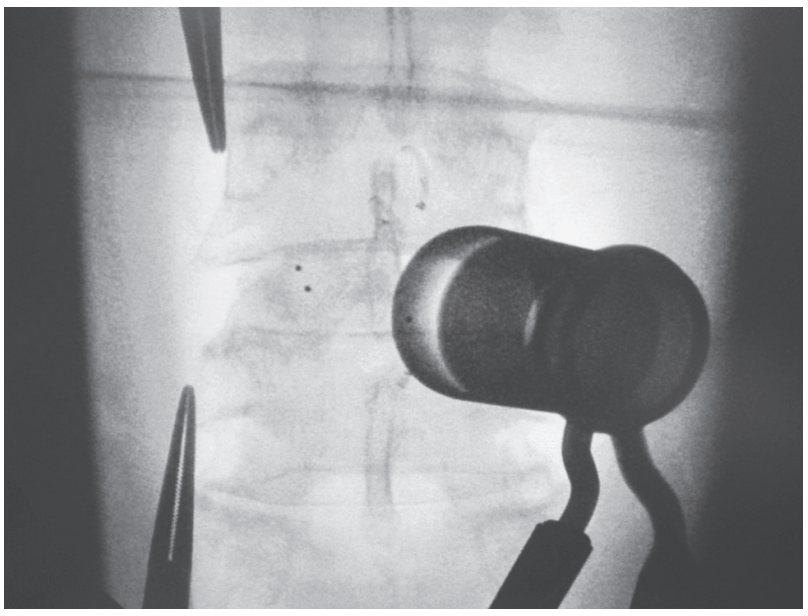

Fig. 3. Anteroposterior fluoroscopic image showing placement of a tubular retractor (right) and landmarks for inserting pedicle screws (left).

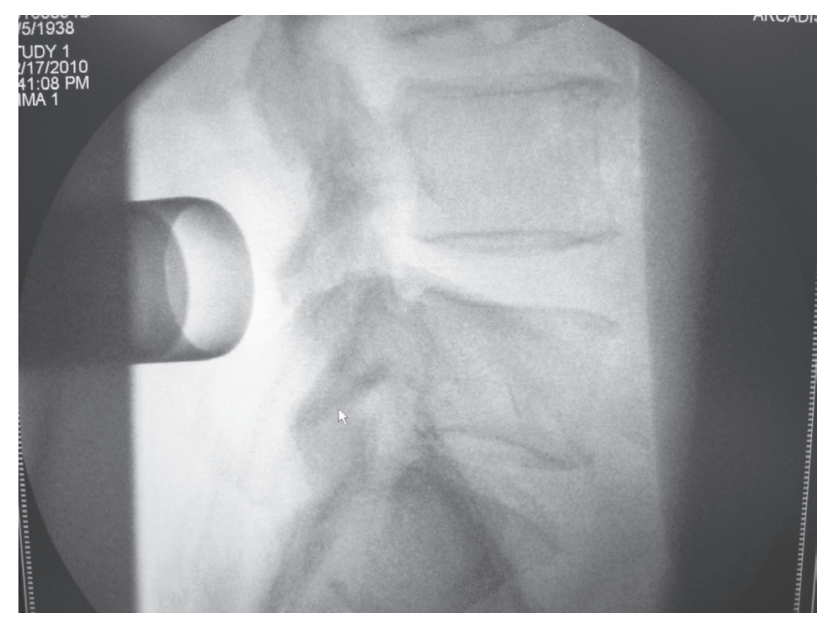

Fig. 4. Lateral fluoroscopic image showing the trajectory of a tubular retractor. 
aspect of the relevant pedicles, and advanced gradually until they were just beyond the base of the pedicle. This was followed by advancing guidewires into the pedicles and vertebral bodies under fluoroscopic guidance. Pedicle screw holes were tapped and screws inserted, and the rods were glided into the screw heads and tightened using set screws under compression (Fig. 5). Pedicle screws ipsilateral to the side of TLIF were inserted using the same incision made for the tubular dilator. The incisions were closed in layers (Fig. 6), and a single drain to be removed on postoperative day 3 or 4 was inserted.

\section{Results}

The mean age for the MI-TLIF cases was 43.6 years (range,

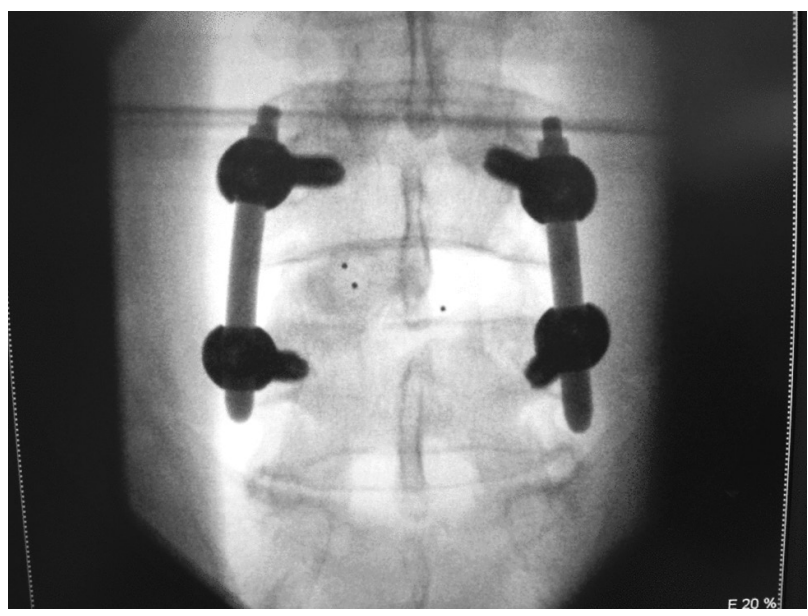

Fig. 5. Anteroposterior fluoroscopic image of lumbar spine post fixation.

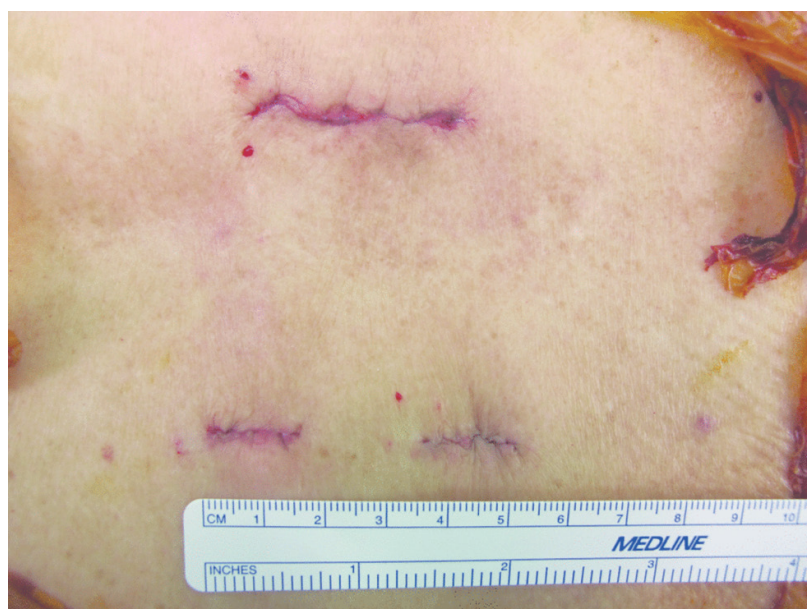

Fig. 6. Clinical picture showing the size of wounds using the minimally invasive approach.
20-69 years) and that for the open cases was 44.4 years (range, 19-69 years). The male: female ratio was 13:12 for both groups. In each group, two patients had degenerative disc disease, 12 had prolapsed intervertebral discs, three had spinal stenosis, and seven had spondylolisthesis. Each group consisted of nine patients who were operated on at L4/5, 12 at L5/S1, and four patients had two levels operated on (L4/5 and L5/S1). Average follow-up was 26.9 months for the MI-TLIF group and 29.3 months for the open group.

The implants used in the MI-TLIF group were the Viper (DePuy Spine, Raynham, MA, USA) screw system and the Concorde (DePuy Spine) cage. Pedicle screw implants used in the open group included 17 Expedium (DePuy Spine), four Monarch (DePuy Spine), two Pangea (Synthes Spine, West Chester, PA, USA), and two CD Horizon Legacy (Medtronic Sofamor Danek, Minneapolis, MN, USA) screw systems. The interbody cages used included 15 Concorde (DePuy Spine), seven Leopard (DePuy Spine), two Travios (Synthes Spine), and one Capstone (Medtronic Sofamor Danek) cage. All Viper screws were $6 \mathrm{~mm}$ in diameter, the Expedium screws were $6 \mathrm{~mm}$ in diameter, the Monarch screws were 6.25 $\mathrm{mm}$ in diameter, the Pangea screws were $6 \mathrm{~mm}$ in diameter, and the CD Horizon Legacy screws were $6.5 \mathrm{~mm}$ in diameter.

Perioperative results are shown in Table 1.

The duration of surgery was significantly longer in the MI-TLIF than that in the open TLIF group. The mean duration of surgery for the open and MI groups was 166.4 minutes vs. 253.9 minutes for L4/5, 191.3 minutes vs. 271.6 minutes for L5/S1, and 252.5 minutes vs. 366.3 minutes for L4 to S1 (all $p<0.05$ ). Blood loss was assessed based on a gross intraoperative estimation and the postoperative pre-transfusion decrease in hemoglobin. Two patients with double level MI-TLIF had blood transfusions prior to the postoperative hemoglobin check. The lengths of stay for both MI and open single level TLIF were similar. However, length of stay was longer for the two level MI-TLIF group than that in the open group $(p<0.05)$. Time to ambulation was similar in both groups, regardless of the number of levels.

A significant improvement in ODI scores was observed at 6 months, 1 year, and 2 years compared to pre-surgery in both groups $(p<0.05)$. However, no significant difference was observed in the ODI scores between the groups. Similar findings were observed for the EQ-5D scores, i.e., 
Table 1. Perioperative results

\begin{tabular}{|c|c|c|}
\hline & Open TLIF & MI-TLIF \\
\hline \multicolumn{3}{|c|}{ Duration of surgery (min) } \\
\hline $\mathrm{L} 4 / 5$ & $166.4(98-245)$ & $253.9(185-350)$ \\
\hline L5/S1 & $191.3(105-315)$ & $271.6(218-340)$ \\
\hline 2-Level (L4-S1) & $252.5(185-285)$ & $366.3(315-405)$ \\
\hline \multicolumn{3}{|c|}{ Estimated intraoperative blood loss (mL) } \\
\hline$\llcorner 4 / 5$ & $238.9(150-350)$ & $222.2(150-400)$ \\
\hline L5/S1 & $237.5(150-400)$ & $233.3(150-300)$ \\
\hline 2-Level (L4-S1) & $267.5(185-320)$ & $362.5(250-400)$ \\
\hline \multicolumn{3}{|c|}{ Drop in haemoglobin (at 1st postoperative day) (g/dL) } \\
\hline$L 4 / 5$ & $2.1(0.5-4.4)$ & $2.2(0.6-3.4)$ \\
\hline L5/S1 & $2.1(0.5-3.6)$ & $2.6(1.6-3.9)$ \\
\hline 2-Level (L4-S1) & $3.0(2.1-4.1)$ & $2.9(2.5-3.1)^{\text {a) }}$ \\
\hline \multicolumn{3}{|c|}{ Length of hospitalization (day) } \\
\hline$L 4 / 5$ & $7.3(5-11)$ & $6.3(5-8)$ \\
\hline L5/S1 & $6.6(5-8)$ & $6.4(5-9)$ \\
\hline 2-Level (L4-S1) & $7.7(6-10)$ & $10.0(7-15)$ \\
\hline \multicolumn{3}{|c|}{ Duration to ambulation (day) } \\
\hline $\mathrm{L} 4 / 5$ & $2.9(2-3)$ & $3.1(2-5)$ \\
\hline $\mathrm{L} 5 / \mathrm{S} 1$ & $2.9(2-5)$ & $3.0(2-4)$ \\
\hline 2-Level (L4-S1) & $3.3(3-4)$ & $3.5(3-5)$ \\
\hline
\end{tabular}

TLIF, transforaminal lumbar interbody fusion; MI-TLIF, minimally invasive transforaminal lumbar interbody fusion.

a) Two patients had blood transfusions of 1 pint of packed red cells each prior to blood taking.

the postoperative improvements at 6 months, 1 year, and 2 years were significant compared to preoperative scores. However, no significant differences were observed in the EQ-5D scores at 6 months, 1 year, or 2 years between the groups.

Interbody fusion rates based on the Bridwell classification were not different between the groups (Table 2). Larger cages were used for open TLIF cases compared to those in the MI-TLIF cases at the L5/S1 level ( $p=0.0134$; 95\% confidence interval, $0.18-1.44$ ). L4/5 fit a larger cage compared with L5/S1 in the MI-TLIF group but this was not a significant difference. No association was detected between cage size, fusion rate, or cage migration at the 2-year follow up. Complications of MI-TLIF and open TLIF are shown in Table 3. Two patients in the MI-TLIF group had residual dermatomal symptoms postoperatively. One showed inadequate decompression of the spinal nerve root on magnetic resonance imaging (MRI). Although four times more complications were
Table 2. Cage height and fusion rates

\begin{tabular}{lccc} 
& Open TLIF & MI TLIF & $p$-value \\
Cage height (mm) & & & \\
Level L4/5 & $10.6(9-13)$ & $10.4(9-12)$ & $>0.05$ \\
Level L5/S1 & $10.6(9-12)$ & $9.8(8-11)$ & $>0.05$ \\
Fusion rates & & & \\
Level L4/5 & $1.5(1-2)$ & $1.9(1-3)$ & $>0.05$ \\
Level L5/S1 & $1.3(1-2)$ & $1.6(1-2)$ & 0.0134 \\
\hline
\end{tabular}

TLIF, transforaminal lumbar interbody fusion; MI-TLIF, minimally invasive transforaminal lumbar interbody fusion.

a'Based on Bridwell fusion grading: Grade I, fused with remodeling and trabeculae; Grade II, graft intact, not fully remodeled and incorporated though; Grade III, graft intact, but a definite lucency at the top or bottom of the graft; Grade IV, definitely not fused with resorption of bone graft and with collapse.

observed in the MI-TLIF group ( $p=0.07)$, it was statistically insignificant. 
Table 3. Complications of MI-TLIF and open TLIF

\begin{tabular}{lcc} 
Complication & MI-TLIF & Open TLIF \\
\hline Non-specific residual lower limb symptoms & $5(20)$ & $2(8)$ \\
\hline Persistent dermatomal pain/numbness ${ }^{\text {a) }}$ & 2 (8) & 0 \\
\hline Deep vein thrombosis/pulmonary embolism & $1(4)$ & 0 \\
\hline Superficial or deep wound infection/haematoma & 0 & 0 \\
\hline Chest infection & 0 & 0 \\
Suboptimal screw placement requiring revision & 0 & 0 \\
\hline Dural tear/nerve root injury & 0 & 0
\end{tabular}

Values are presented as number (\%).

MI-TLIF, minimally invasive transforaminal lumbar interbody fusion; TLIF, transforaminal lumbar interbody fusion.

${ }^{a}$ Magnetic resonance imaging was done for both patients. One patient showed residual spinal stenosis; ${ }^{b}$ Patient had two-level MI-TLIF.

\section{Discussion}

This is one of the few reports that has analyzed intermediate results between MI-TLIF and open TLIF. Studies have been conducted to show the outcomes of MI-TLIF $[8,9]$. However, few prospective studies have actually compared MI-TLIF with open TLIF [10-13]. Another similar study with a larger study population was published very recently [14]. However, no large randomized controlled trials have shown the benefits of MI-TLIF over an open group [6]. Therefore, the potential benefits of MI-TLIF over open TLIF remain controversial. The same authors that proposed the advantages of MI-TLIF often acknowledged the technical challenges they faced operating through a limited corridor [10-13]. Although ours was not a prospective randomized study, it was a well controlled comparative study. We recognize that the cost of surgery could be a potential bias during selection of patients for surgery but this could not be adjusted.

It is not difficult to rationalize that minimally invasive techniques reduce morbidity through smaller skin incisions, less paraspinal muscle dissection, and reduced soft tissue retraction [14-18]. This, in turn, reduces blood loss and alleviates the need for blood transfusion and its associated risks [19-21]. The surgical corridor during open surgery is exceedingly wide and results in substantial degree of soft tissue injury to the paraspinal musculature. Neurovascular structures are often violated during dissection to expose the spine. As shown in a recent literature review by Kim [22], MI-TLIF avoids these problems by opening a narrow surgical corridor directly over the TLIF target site; thus, avoiding injury to the musculoliga- mentous complex and excessive intraoperative bleeding. A multicenter randomized study conducted by Alamin et al. [23] to evaluate the effect of MI-TLIF vs. open TLIF on paraspinal musculature using MRI showed that both quantitative and qualitative measures of edema in the multifidus were significantly less in the MI-TLIF group, which is consistent with less muscle injury.

Minimally invasive methods used in TLIF include the mini-open approach and the MI-TLIF technique. The former approach evolved from the original paraspinal bilateral muscle splitting exposure first described by Wiltse et al. [24]. This approach involves bilateral paraspinal sacrospinalis muscle-splitting using expandable tubular retractors, whereas MI-TLIF involves unilateral paraspinal sacrospinalis muscle-splitting using expandable tubular retractors with percutaneous screw placement on the opposite side. Drawbacks of MI-TLIF include the limited ability to visualize certain anatomic landmarks and reliance on fluoroscopy. Mini-open approaches conceptually combine the advantages of traditional open surgery (anatomic visualization and ability to palpate instrumentation trajectories) [25] and the advantages of an MI approach (reduced tissue trauma). One study [10] showed a significant decrease in blood loss and length of hospital stay in a mini-open group compared to those in an open TLIF group.

In our study, patients in both groups underwent similar postoperative management. The decrease in hemoglobin, duration required to achieve ambulation, and length of hospital stay were comparable for single level TLIFs in both groups. However, two level MI-TLIFs had greater blood loss and required a longer duration to ambulate 
and be discharged. This was associated with a longer operative time. As MI-TLIF is technically more demanding, we recognize that this could result in longer operative time [10,26], greater intraoperative blood loss [10], delayed ambulation, and lower fitness for discharge.

Various factors may have contributed to the prolonged operative time of our MI-TLIF approach. First, surgeons are required to operate through limited exposure and are forced to maneuver in less space through the tubular dilator retractors. Second, better coordination between the surgeon and assistant is necessary to maintain a bloodless field during surgery. Last, as with all minimally invasive surgical strategies, this technique is associated with a steep learning curve. This was particularly true here, as our MI-TLIF cases represented the earlier cases performed by the surgeon. We believe that as surgeons become more versatile with MI-TLIF, a gradual reduction in both operative time and blood loss is expected [10].

In our study, smaller-sized cages used for MI-TLIF at the L5S1 level could be secondary to the challenges faced when inserting a cage through tubular retractors. The surgeon's ability to insert the most appropriately sized cage could have been compromised, resulting in inserting a smaller cage. Unlike open TLIF, the inability to use a distraction device to facilitate placement of the interbody cage during MI-TLIF may further add to the possibility of an undersized cage being placed. This is particularly true at the L5/S1 level due to the relatively awkward trajectory during cage insertion. One possible option to overcome this is to insert contralateral pedicle screws first, and subsequently distract the screws to allow some degree of distraction of the disc space to aid in placing the cage. However, this should be used judiciously in patients with osteoporotic bones, for fear of causing premature loosening of screws.

One important complication seen in our series of patients who underwent MI-TLIF included a patient with a deep vein thrombosis leading to a pulmonary embolism. This has not been documented previously in patients undergoing MI-TLIF. This patient was obese (body mass index $>35 \mathrm{~kg} / \mathrm{m}^{2}$ ) and who had previous decompression at $\mathrm{L} 4 / 5$ and L5/S1. It is likely that the prolonged surgery caused venous stasis in the lower limbs despite the routine use of intraoperative and postoperative calf pumps in all of our patients. This patient required treatment with clexane, which was later converted to warfarin. The patient has since recovered from this condition and was well at the 2 year follow-up. No patient developed a wound infection, which was consistent with the findings of Rovner et al. [27] who found no patient who underwent MI-TLIF developed a wound infection in his retrospective series of 196 patients. Although two patients in our study had persistent residual dermatomal pain and numbness, only one showed residual stenosis at the TLIF level, suggesting inadequate decompression on MRI. That patient improved after physiotherapy and acupuncture, alleviating the need for further surgery. We believe that these symptoms were contributed partly by the technical difficulties at decompression, and partly by inadequate distraction due to insertion of a smaller intervertebral cage at the L5S1 level. This might be amenable via experience and frequent use of "over the top" decompression. Potential worsening of neurological status was also demonstrated by De La Torre et al. [28] who found a prevalence of 3\%. That study had one morbidly obese patient with an interbody graft failure, and two patients who required re-positioning of the interbody graft. With such MI-TLIF complications seen in various studies, including ours, the selection of MITLIF candidates should be very stringent. Prevention and early detection of complications are essential to avoid poor outcomes.

In our study, the satisfactory results for both MI-TLIF and open TLIF with a minimum 2 year follow-up suggest that either surgical technique can be safely and effectively used in appropriately selected patients. In accordance with other studies, we believe that fusion is an important determining factor in the functional improvement of our patients $[12,13]$. This was reflected in the improved ODI functional scores for all of our patients at the 6 month, 1 year, and 2 year follow-ups. A study by Hall and Mobasser [29] on 21 patients undergoing MI-TLIF showed that unilateral pedicle screw instrumentation provides adequate stability for lumbar interbody fusion. However, with the lack of further studies on larger populations, this result should be interpreted with caution. No study during the conduct of this study has been performed to evaluate the effect of bone morphogenetic protein (BMP) on fusion rates after TLIF surgeries. A meta-analysis of MI-TLIF (BMP used in 50\%) and open TLIF (BMP used in $12 \%$ ) showed relatively high and similar fusion rates between the two groups [30]. In our study, we routinely used interbody cages packed with local autogenous bone graft.

Given the comparable long-term functional outcomes 
of open and MI-TLIF, patients requiring TLIF are pursuing the cosmetically nicer scars offered by MI-TLIF. We recommend that patients be informed of the potentially longer operations, greater blood loss, and longer hospitalization when there is a need to perform multiple-level TLIF. This is attributed to the technical difficulties while operating through a limited area and can be made worse in the hands of an unprepared surgeon.

\section{Conclusions}

MI-TLIF and open TLIF had comparable short-term results in terms of estimated blood loss, duration to ambulation, and length of hospital stay. Intermediate-term fusion rate results and patient-reported outcome scores at 2 years were also comparable in both surgical procedures.

Although previous studies have shown that the paraspinal musculature is better preserved using the MI technique, its technical constraints coupled with a steep learning curve may result in longer operative time and potential undersizing of cages at the L5S1 level. Moreover, a multi-level MI-TLIF procedure may result in a longer hospitalization stay.

\section{Conflict of Interest}

No potential conflict of interest relevant to this article was reported.

\section{Acknowledgments}

We would like to acknowledge help from the following: 1) Dr. Chan Yiong Huak for his help in statistical analysis and calculation. 2) Ms. Heng Wee May for her help in the collection and organization of data. 3) Dr. Ouyang Youheng for his help in the collection of data. 4) Dr. Danakkrisna Vachalam for his help in the submission process.

\section{References}

1. Harms J, Rolinger H. A one-stager procedure in operative treatment of spondylolistheses: dorsal traction-reposition and anterior fusion (author's transl). Z Orthop Ihre Grenzgeb 1982;120:343-7.

2. Cole CD, McCall TD, Schmidt MH, Dailey AT. Comparison of low back fusion techniques: transforaminal lumbar interbody fusion (TLIF) or posterior lumbar interbody fusion (PLIF) approaches. Curr Rev Musculoskelet Med 2009;2:118-26.

3. Lin PM. Posterior lumbar interbody fusion technique: complications and pitfalls. Clin Orthop Relat Res 1985;(193):90-102.

4. Brantigan JW, Steffee AD, Lewis ML, Quinn LM, Persenaire JM. Lumbar interbody fusion using the Brantigan I/F cage for posterior lumbar interbody fusion and the variable pedicle screw placement system: two-year results from a Food and Drug Administration investigational device exemption clinical trial. Spine (Phila Pa 1976) 2000;25:1437-46.

5. Asano S, Kaneda K, Umehara S, Tadano S. The mechanical properties of the human L4-5 functional spinal unit during cyclic loading. The structural effects of the posterior elements. Spine (Phila Pa 1976) 1992;17:1343-52.

6. Hey HW, Hee HT. Lumbar degenerative spinal deformity: Surgical options of PLIF, TLIF and MI-TLIF. Indian J Orthop 2010;44:159-62.

7. Bridwell KH, Lenke LG, McEnery KW, Baldus C, Blanke K. Anterior fresh frozen structural allografts in the thoracic and lumbar spine. Do they work if combined with posterior fusion and instrumentation in adult patients with kyphosis or anterior column defects? Spine (Phila Pa 1976) 1995;20:1410-8.

8. Park P, Foley KT. Minimally invasive transforaminal lumbar interbody fusion with reduction of spondylolisthesis: technique and outcomes after a minimum of 2 years' follow-up. Neurosurg Focus 2008;25:E16.

9. Deutsch H, Musacchio MJ Jr. Minimally invasive transforaminal lumbar interbody fusion with unilateral pedicle screw fixation. Neurosurg Focus 2006; 20:E10.

10. Dhall SS, Wang MY, Mummaneni PV. Clinical and radiographic comparison of mini-open transforaminal lumbar interbody fusion with open transforaminal lumbar interbody fusion in 42 patients with longterm follow-up. J Neurosurg Spine 2008;9:560-5.

11. Schizas C, Tzinieris N, Tsiridis E, Kosmopoulos V. Minimally invasive versus open transforaminal lumbar interbody fusion: evaluating initial experience. Int Orthop 2009;33:1683-8.

12. Shunwu F, Xing Z, Fengdong Z, Xiangqian F. Minimally invasive transforaminal lumbar interbody fusion for the treatment of degenerative lumbar diseases. Spine (Phila Pa 1976) 2010;35:1615-20. 
13. Peng CW, Yue WM, Poh SY, Yeo W, Tan SB. Clinical and radiological outcomes of minimally invasive versus open transforaminal lumbar interbody fusion. Spine (Phila Pa 1976) 2009;34:1385-9.

14. Lee KH, Yue WM, Yeo W, Soeharno H, Tan SB. Clinical and radiological outcomes of open versus minimally invasive transforaminal lumbar interbody fusion. Eur Spine J 2012;21:2265-70.

15. Gejo R, Matsui H, Kawaguchi Y, Ishihara H, Tsuji H. Serial changes in trunk muscle performance after posterior lumbar surgery. Spine (Phila Pa 1976) 1999; 24:1023-8.

16. Rantanen J, Hurme M, Falck B, et al. The lumbar multifidus muscle five years after surgery for a lumbar intervertebral disc herniation. Spine (Phila $\mathrm{Pa}$ 1976) $1993 ; 18: 568-74$.

17. Sihvonen T, Herno A, Paljarvi L, Airaksinen O, Partanen J, Tapaninaho A. Local denervation atrophy of paraspinal muscles in postoperative failed back syndrome. Spine (Phila Pa 1976) 1993;18:575-81.

18. Styf JR, Willen J. The effects of external compression by three different retractors on pressure in the erector spine muscles during and after posterior lumbar spine surgery in humans. Spine (Phila Pa 1976) 1998; 23:354-8.

19. Hee HT, Castro FP Jr, Majd ME, Holt RT, Myers L. Anterior/posterior lumbar fusion versus transforaminal lumbar interbody fusion: analysis of complications and predictive factors. J Spinal Disord 2001; 14:533-40.

20. Humphreys SC, Hodges SD, Patwardhan AG, Eck JC, Murphy RB, Covington LA. Comparison of posterior and transforaminal approaches to lumbar interbody fusion. Spine (Phila Pa 1976) 2001;26:567-71.

21. Karikari IO, Isaacs RE. Minimally invasive transforaminal lumbar interbody fusion: a review of techniques and outcomes. Spine (Phila Pa 1976) 2010;35:
S294-301.

22. Kim CW. Scientific basis of minimally invasive spine surgery: prevention of multifidus muscle injury during posterior lumbar surgery. Spine (Phila Pa 1976) 2010;35:S281-6.

23. Alamin T, Hardenbrook M, Kim C, et al. 17. Preliminary results of a multicenter randomized study to evaluate the effect of minimally invasive vs. open lumbar fusion on paraspinal musculature using MR imaging. Spine J;8:8S-9S.

24. Wiltse LL, Bateman JG, Hutchinson RH, Nelson WE. The paraspinal sacrospinalis-splitting approach to the lumbar spine. J Bone Joint Surg Am 1968;50:919-26.

25. Mummaneni PV, Rodts GE Jr. The mini-open transforaminal lumbar interbody fusion. Neurosurgery 2005;57:256-61.

26. Perez-Cruet M, Tobler W, Lennarson P, McCafferty R, Spoonamore M, Araghi A. P131. Early results of a prospective, multicenter, randomized clinical trial evaluating minimally invasive and open pedicle screw implantation outcomes. Spine J 2005;5:S173.

27. Rovner J, Schwender J, Mullaney K, Pinto M, Garvey T. 19. A comparison of infection rates in minimally invasive vs. open TLIFs: a single surgeon retrospective review. Spine J;8:9S-10S.

28. De La Torre RP, Mishkoor K, Chabot J, Perez-Cruet M. P95. Minimally invasive transforaminal lumbar interbody fusion and percutaneous pedicle screw fixation long-term outcomes. Spine J;8:146S-147S.

29. Hall G, Mobasser JP. P15. Unilateral pedicle screw instrumentation in minimally invasive lumbar fusion. Spine J;8:108S-109S.

30. Wu RH, Fraser JF, Hartl R. Minimal access versus open transforaminal lumbar interbody fusion: metaanalysis of fusion rates. Spine (Phila Pa 1976) 2010; 35:2273-81. 